Pesq. Vet. Bras. 37(10):1085-1090, outubro 2017 DOI: $10.1590 / \mathrm{S} 0100-736 \mathrm{X} 2017001000008$

\title{
Distribuição espacial e abundância de carrapatos (Acari: Ixodidae) em remanescente de Mata Atlântica, Nordeste do Brasil ${ }^{1}$
}

\author{
Cristina F. da Fonseca², Débora C.V. de Lima², Dênisson da S. e Souza², Silvia G.N. \\ da Silva ${ }^{2}$, Jaciara R.B. de Lima ${ }^{4}$, Jaqueline B. de Oliveira ${ }^{2}$, Geraldo J.B. de Moura ${ }^{2}$ \\ e Filipe M. Aléssio ${ }^{3 *}$
}

\begin{abstract}
Fonseca C.F., Lima D.C.V., Souza D.S., Silva S.G.N., Lima J.R.B., Oliveira J.B., Moura G.J.B. \& Aléssio F.M. 2017. [Spatial distribution and abundance of ticks (Acari: Ixodidae) in Atlantic forest remaining, northeastern Brazil.] Distribuição espacial e abundância de carrapatos (Acari: Ixodidae) em remanescente de Mata Atlântica, Nordeste do Brasil. Pesquisa Veterinária Brasileira 37(10):1085-1090. Instituto de Ciências Biológicas, Universidade de Pernambuco, Rua Arnóbio Marques 310, Santo Amaro, Recife, PE 50100130, Brazil. E-mail: filipe.alessio@gmail.com

The spatial distribution and abundance of free-living ticks were examined in Atlantic Forest fragments in northeastern Brazil. The study was conducted on Integral Protection Conservation Units of Mata do Tapacurá and Mata do Camucim, located in the municipality of São Lourenço da Mata, Pernambuco. Ticks were captured by flagging method in six plots of $200 \mathrm{~m}^{2}$, three plots set in a forest edge adjacent to an agropastoral matrix and three plots set in the forest edge adjacent to a dam lake. Were captured 2652 ticks of the genus Amblyomma, two nymphs identified as Amblyomma dissimile with spatially aggregated distribution, shown by the variance/mean ratio and Lloyd aggregate index. There was significant variation in the abundance of ticks between plots $(\mathrm{H}=26.79, \mathrm{p}<0.005)$ and more ticks was found in the woods adjacent to the edge agropastoral matrix. There was a positive correlation between canopy cover and tick density ( $\mathrm{rs}=0.31, \mathrm{p}<0.05$ ). In the present study, it was observed that in free-living stages, especially larvae and nymphs, the spatial distribution and abundance of ticks are strongly influenced by more enclosed areas of forests. Most ticks near agropastoral matrix could facilitate the migration of ticks between wild environments and man-made or modified environments.
\end{abstract}

INDEX TERMS: Ticks, Acari, Ixodidae, Atlantic forest, northeastern Brazil, Amblyomma, Amblyomma dissimile, dispersion indexes, microhabitat, environmental descriptors.

RESUMO.- A distribuição espacial e a abundância de carrapatos em estágio de vida livre foram examinadas em fragmentos de Mata Atlântica no Nordeste do Brasil. 0 estudo foi realizado em Unidades de Conservação de Proteção Integral Mata do Tapacurá e Mata do Camucim, localizadas no município de São Lourenço da Mata, Pernambuco. Os

\footnotetext{
${ }^{1}$ Recebido em 31 de julho de 2016.

Aceito para publicação em 22 de março de 2017.

${ }^{2}$ Departamento de Biologia, Universidade Federal Rural de Pernambuco (UFRPE), Rua Dom Manoel de Medeiros s/n, Dois Irmãos, Recife, PE 52171-900, Brasil.

${ }^{3}$ Instituto de Ciências Biológicas, Universidade de Pernambuco (UPE), Rua Arnóbio Marques 310, Santo Amaro, Recife, PE 50100-130, Brasil. *Autor para correspondência: filipe.alessio@gmail.com

${ }^{4}$ Universidade do Estado da Bahia (UNEB), Rua Silveira Martins 2555, Cabula, Salvador, BA 41150-000, Brasil.
}

carrapatos foram capturados pelo método da bandeira de flanela em seis parcelas de $200 \mathrm{~m}^{2}$, sendo três parcelas estabelecidas em uma borda de mata adjacente a uma matriz agropastoril e outras três parcelas estabelecidas na borda de mata adjacente ao lago de barragem. Foram capturados 2652 carrapatos do gênero Amblyomma, sendo duas ninfas identificadas como Amblyomma dissimile com distribuição espacialmente agregada, demonstrados pela relação variância/média e o índice de agregação de Lloyd. Houve variação significativa na abundância de carrapatos entre as parcelas $(H=26,79, p<0,005)$ sendo encontrados mais carrapatos na borda da mata adjacente à matriz agropastoril. Quanto as variáveis ambientais, houve correlação positiva entre a cobertura de dossel e a densidade de carrapatos $(\mathrm{rs}=0,31, \mathrm{p}<0,05)$. No presente estudo observou-se que em estágios de vida livre, notadamente larvas e ninfas, a 
distribuição espacial e a abundância de carrapatos são fortemente influenciadas por áreas mais fechadas das matas. A maior presença de carrapatos próximos à matriz agropastoril poderia facilitar a migração de carrapatos entre ambientes silvestres e ambientes antrópicos.

TERMOS DE INDEXAÇ̃̃O: Carrapatos, Acari, Ixodidae, Mata Atlântica, Nordeste do Brasil, Amblyomma, Amblyomma dissimile, índices de dispersão, microhabitat, descritores ambientais.

\section{INTRODUÇÃO}

O Brasil possui uma grande variedade de carrapatos, compreendendo cerca de 66 espécies (Martins et al. 2014, Nava et al. 2014), onde o gênero Amblyomma Koch, 1844 engloba a maior diversidade de espécies (Aragão 1936) e possui a maior amplitude de hospedeiros relacionados (Anderson \& Magnarelli 1993), sendo representada por 130 espécies (Nava et al. 2009), das quais 30 são estabelecidas no Brasil (Dantas-Torres et al. 2009). A maioria dos estudos envolve a fase adulta dos carrapatos, enquanto as fases imaturas ainda são pouco conhecidas principalmente na fase de vida livre em ambientes naturais (Labruna et al. 2005, Szabó et al. 2007, 2009). Os padrões de distribuição espacial e de abundância de carrapatos são relevantes para a conservação da biodiversidade, detectando as condições ambientais favoráveis à manutenção desses vetores e consequentemente as doenças potencialmente transmitidas (Horta et al. 2011).

Neste contexto, o bioma Mata Atlântica destaca-se por apresentar ampla diversidade de fauna e flora (Galindo-Leal \& Câmara 2003, Silvano \& Segalla 2005), sendo a vegetação utilizada como suporte de contato entre carrapatos e possíveis hospedeiros (Szabó et al. 2009), como sítio de oviposição, substrato para a muda e proteção contra o calor (Guerra et al. 2002). Devido aos impactos antrópicos, a distribuição da Mata Atlântica na costa brasileira encontra-se fragmentada e fortemente urbanizada (Galindo-Leal \& Câmara 2003), havendo maior contato entre a vida selvagem e seus patógenos, ampliando o ciclo de transmissibilidade de doenças por carrapatos aos animais domésticos e ao homem (Allan et al. 2003, Silveira et al. 2007, Spolidorio et al. 2010, Dantas-Torres et al. 2012).

Segundo Murcia (1995) e Rodrigues (1998), os fragmentos florestais implicam no surgimento de uma borda ou área de transição entre as pressões antrópicas e o fragmento florestal, com influências físicas e características de microambiente diferenciadas do centro e que podem influenciar negativamente no equilíbrio ecológico, e neste caso na dispersão dos estádios imaturos dos carrapatos no ambiente.

O aumento de atividades antrópicas como a pecuária, pesquisas e o ecoturismo nas áreas de conservação ambiental pode ser um risco para a ocorrência de zoonoses transmitidas por carrapatos (Pinheiro et al. 2014), considerando que mais de $90 \%$ do tempo do ciclo de vida desses vetores ocorre no ambiente à espera de hospedeiros (Sonenshine et al. 2002). Nos estágios de vida livre, notadamente larvas e ninfas, a distribuição espacial, abundância e consequentemente densidade de carrapatos são influenciadas por fatores abióticos e bióticos, tais como tempera- tura, umidade, vegetação e disponibilidade de hospedeiros (Randolph \& Storey 1999, Lindgren et al. 2000, Estrada-Penã 2001, Schulze \& Jordan 2005, Peterka 2008, Aléssio et al. 2012).

Nesse sentido, objetivou-se investigar a distribuição espacial, abundância e densidade de carrapatos em fragmento de Mata Atlântica, detectando quais descritores ambientais melhor explicam os parâmetros ecológicos registrados.

\section{MATERIAL E MÉTODOS}

Área de estudo. 0 estudo foi realizado nas Unidades de Conservação de Proteção Integral, Refúgios de Vida Silvestre Mata do Camucim (40,24 Ha) e Engenho Tapacurá (316,32 Ha), composto por remanescentes de Mata Atlântica, situados no Município de São Lourenço da Mata, Zona da Mata do estado de Pernambuco (82' 12" S, 35 11' 41" W) (Fig.1) (Pernambuco 2011). A matriz que circunda as Unidades de Conservação é constituída por um mosaico irregular de monocultura de cana de açúcar, atividades agropastoris e vegetação nativa em regeneração e ao sul, os remanescentes de mata são margeados pelo lago da barragem Tapacurá.

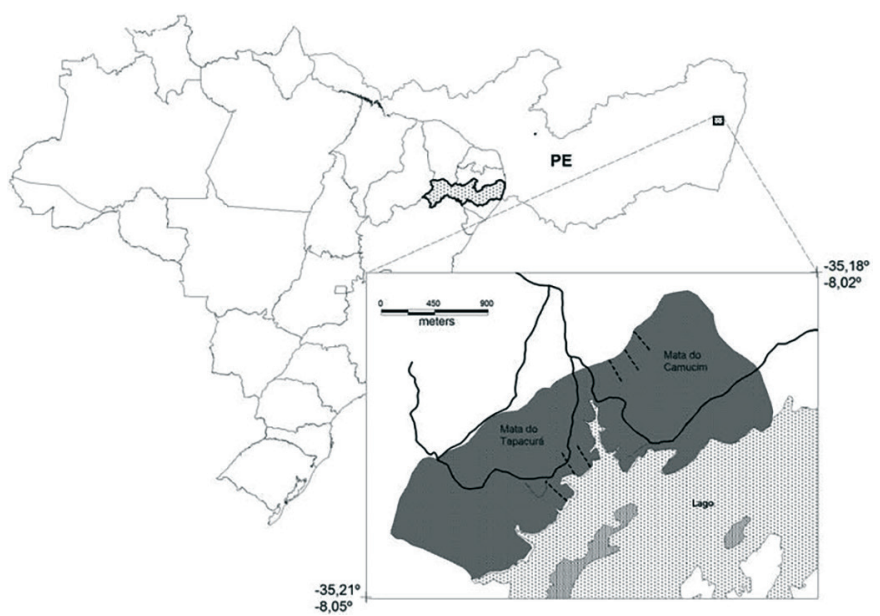

Fig.1. Área de estudo, destacando as Unidades de Conservação de Proteção Integral Refúgios de Vida Silvestre Mata do Camucim e do Engenho Tapacurá, estado de Pernambuco, Brasil $\left(8^{\circ} 2^{\prime}\right.$ $12^{\prime \prime} \mathrm{S}, 35^{\circ} 11^{\prime} 41^{\prime \prime} \mathrm{W}$ ), destacando, em pontilhado, as parcelas de amostragem.

Os fragmentos são classificados como Mata Estacional Semidecidual, com média pluviométrica anual de $1300 \mathrm{~mm}$, apresentando cinco meses secos que vão de setembro a janeiro (Condepe 2000). A vegetação de mata seca é predominantemente arbórea, atingindo cerca de $30 \mathrm{~m}$ de altura (Lyra-Neves et al. 2007).

Método. Os dados foram coletados ao longo de seis parcelas de $200 \mathrm{~m}^{2}$ estabelecidas perpendicularmente em relação às bordas dos fragmentos estudados, sendo três na borda sul da Mata do Tapacurá adjacente à margem do lago Tapacurá e três localizados na borda noroeste da Mata do Camucim, adjacente à região de matriz agropastoril (Fig.1). As coletas foram realizadas no mês abril de 2016 por meio de varreduras com auxílio de bandeiras confeccionadas em tecido branco de algodão medindo $75 \mathrm{~cm}$ de comprimento e $60 \mathrm{~cm}$ de altura, presas em haste de madeira, conforme protocolo adaptado de Rulison et al. (2013). Cada parcela de $200 \mathrm{~m}^{2}$ foi vistoriada três vezes. As bandeiras foram examinadas a cada dez metros longitudinais a parcela e os carrapatos encontrados colocados em tubos de tipo eppendorf com álcool isopropílico. As vistorias foram realizadas por duas pessoas amostrando os 
lados direito e esquerdo até um metro de distância do centro da parcela, perfazendo um total de $3600 \mathrm{~m}^{2}$ vistoriados.

Os carrapatos foram identificados utilizando as chaves de Onofrio et al. (2006) e Martins et al. (2010), no Laboratório de Parasitologia (LAPAR) da Universidade Federal Rural de Pernambuco (UFRPE).

Quanto à caracterização de microhabitat, as parcelas foram segmentadas em dez subparcelas de $10 \times 2 \mathrm{~m}$, seguindo o método adaptado de Freitas et al. (2002). Em cada subparcelas foram estabelecidas duas estações de coleta de dados espaçadas cinco metros entre si. Para as medições foi utilizado um quadro de madeira de $50 \times 50 \mathrm{~cm}$ entrelaçado por fios de nylon formando cem quadrados vazados. Em cada estação foram medidas, em porcentagem, as seguintes variáveis: cobertura de plantas vivas ao nível do solo, cobertura de serapilheira e cobertura de pedras através do quadro de madeira na posição horizontal apontado para baixo; cobertura de dossel com o quadro de madeira na posição horizontal apontado para cima; e porcentagem do índice de densidade de plantas (obstrução vertical) com o quadro de madeira apontado na posição vertical a uma altura de $50 \mathrm{~cm}$ para os quatro pontos cardeais (para mais detalhes, ver Freitas et al. 2002).

Todos os dados foram testados quanto à normalidade através do teste Shapiro-Wilk no programa PAST. 0 teste não paramétrico Kruskal-Wallis foi realizado para verificar diferenças nos números de carrapatos entre os tipos de borda. Objetivando testar a influência dos descritores ambientais sobre a distribuição espacial, abundância e densidade dos carrapatos, devido a discrepâncias dos dados, se fez necessário extrair à raiz quadrada dos dados absolutos para posterior aplicação do teste de correlação de Spearman. Os índices de dispersão foram calculados com o programa PASSaGE 2 (Rosenberg \& Anderson 2011).

\section{RESULTADOS}

Em total, foram coletadas 2650 larvas e duas ninfas do gênero Amblyomma, sendo realizada a identificação morfológica até o nível de espécie nas duas ninfas que foram caracterizadas como Amblyomma dissimile Koch, 1844 (Quadro $1)$.

O número médio de carrapatos encontrados por parcela com presença de carrapatos foi 69,8 (IC=89,8; $p=0,05)$. 0 intervalo de confiança elevado indica que os carrapatos podem apresentar uma distribuição espacial agregada (Rózsa et al. 2000).

A relação variância/média e o índice de agregação de Lloyd para cada parcela indica a característica agregada da distribuição de Amblyomma sp. (Quadro 2), em que valores maiores que 1 indicam distribuição agregada em ambos os

\section{Quadro 1. Densidade de carrapatos do gênero Amblyomma por parcela nas Unidades de Conservação de Proteção Integral, Pernambuco, Brasil}

\begin{tabular}{|c|c|c|c|c|c|c|c|c|c|c|c|c|}
\hline \multirow[t]{2}{*}{ Borda } & \multirow{2}{*}{$\begin{array}{l}\text { Parcelas } \\
\left(200 \mathrm{~m}^{2}\right)\end{array}$} & \multicolumn{10}{|c|}{ Subparcelas $\left(20 \mathrm{~m}^{2}\right)$} & \multirow[b]{2}{*}{ Total } \\
\hline & & 1 & 2 & 3 & 4 & 5 & 6 & 7 & 8 & 9 & 10 & \\
\hline Agropastoril & A & 30 & 3 & 2 & 8 & 15 & 2 & 5 & 11 & 1701 & $476^{*}$ & 2253 \\
\hline (Mata do & B & 0 & 38 & 1 & 0 & 2 & 3 & 1 & 1 & 0 & 0 & 46 \\
\hline Camucim) & $\mathrm{C}$ & 1 & 12 & 23 & 9 & 64 & 8 & 43 & $19 *$ & 17 & 31 & 227 \\
\hline Lago do Tapacurá & rá D & 1 & 6 & 2 & 0 & 1 & 0 & 0 & 4 & 0 & 2 & 16 \\
\hline (Mata do Engenho & ho E & 0 & 0 & 0 & 0 & 5 & 0 & 0 & 0 & 0 & 0 & 5 \\
\hline $\begin{array}{l}\text { de Tapacurá) } \\
\text { Total }\end{array}$ & $\mathrm{F}$ & 30 & 0 & 9 & 1 & 35 & 0 & 0 & 0 & 0 & 30 & $\begin{array}{c}105 \\
2652\end{array}$ \\
\hline
\end{tabular}

* Presença de uma ninfa na subparcela.
Quadro 2. Distribuição espacial de carrapatos do gênero Amblyomma em Unidades de Conservação de Proteção Integral, Pernambuco, Brasil

\begin{tabular}{ccc}
\hline Parcela & Relação variância/média & Índice de Lloyd \\
\hline A & $1289^{*}$ & 6,71 \\
B & $30,15^{*}$ & 7,33 \\
C & $15,77^{*}$ & 1,65 \\
D & 2,52 & 1,9 \\
E & $5^{*}$ & 9 \\
F & $21,2^{*}$ & 2,92
\end{tabular}

* Diferenças significativas $(\mathrm{p}<0,05)$ em relação à distribuição aleatória.

índices, A distribuição agregada não foi significativamente diferente de uma distribuição uniforme para a parcela D.

Na subparcela A9 foram coletados $64,1 \%(n=1701)$ de todos os carrapatos do estudo. A densidade média total observada foi 44,2 carrapatos por $200 \mathrm{~m}^{2}$. Dos 60 trechos amostrados, $63,3 \%(n=38)$ apresentaram 1 ou mais carrapatos. A densidade máxima registrada foi de 85,05 carrapatos $/ \mathrm{m}^{2}$.

A variação no número de carrapatos coletados entre as parcelas foi significativamente diferente (análise de variância Kruskal-Wallis $\mathrm{H}=26,79 ; \mathrm{p}<0,005)$. As parcelas da borda do açude (Mata do Engenho de Tapacurá) apresentaram vinte vezes menos carrapatos que as parcelas localizadas na borda da matriz agrícola (Mata do Camucim).

Quanto às variáveis ambientais, só houve correlação positiva entre a cobertura de dossel e a densidade de carrapatos ( $r s=0,31 ; p<0,05)$.

\section{DISCUSSÃO}

No presente estudo foi observada uma maior quantidade de larvas e ninfas em relação aos adultos, o que está de acordo com os estudos realizados por Barreira et al. (2014) e Ramos et al. (2014). Apesar de não poderem ser identificadas em nível de espécie, as larvas pertenciam ao gênero Amblyomma, sendo duas espécies já identificadas ocorrendo nessas áreas, Amblyomma fuscum (Dantas-Torres et al. 2012, Aléssio et al. 2012) e Amblyomma rotundatum (Santos et al. 2002, Dantas-Torres et al. 2008). A espécie Amblyomma dissimile é comumente encontrada em anfíbios e répteis e apresenta ampla distribuição em vários países das Américas, incluindo alguns estados brasileiros (Acre, Amazonas, Pará, Roraima, Pernambuco, Mato Grosso do Sul, Mato Grosso e São Paulo) (Aragão 1936, Schumaker \& Barros 1994, Onofrio 2007) sendo registrada nesta região do município de São Lourenço da Mata, Pernambuco, parasitando lagartos da espécie Tropidurus hispidus (Dantas-Torres et al. 2008).

No presente estudo, foram capturados 2652 carrapatos. Pinheiro et al. (2014) capturaram 152 espécimes de carrapatos ao longo de onze meses através de diferentes métodos de amostragem um fragmento de Mata Atlântica no Estado do Rio de Janeiro, enquanto Szabó et al. (2009), em quatro anos de estudo, colheram 1677 carrapatos, entre ninfas e adultos e 70 agrupamentos de larvas de sete espécies diferentes, apesar de cada agrupamento de larvas poder conter centenas ou milhares de indivíduos. Se a contagem de larvas for realizada, o número de indivíduos capturados ao longo de um estudo sistemático em regi- 
ões heterogêneas, incluindo fragmentos de Mata Atlântica, pode ser bastante elevado, como observado no trabalho de Silveira \& Fonseca (2013) no qual foram capturados mais de 64 mil carrapatos ao longo de quatro anos de coleta.

Pinheiro et al. (2014) discutem que a metodologia de arrasto de flanela não é muito indicada para estudos de amostragem de carrapatos em trilhas em ambientes de Mata Atlântica, pois a vegetação muitas vezes fechada das trilhas pode dificultar a passagem da flanela. A escolha do método de varredura da vegetação por meio de bandeira, se comparado com o arrasto de flanela, talvez seja mais apropriada para estudos realizados em ambientes de vegetação mais densa. A busca visual e armadilhas de $\mathrm{CO}_{2}$ podem ser metodologias bastante eficazes na Mata Atlântica.

No presente estudo, a razão entre variância e a média e o índice de Lloyd demonstraram que as larvas de Amblyomma apresentam uma distribuição agregada e, conforme Daniels \& Fish (1990) e Ostfeld et al. (1996), de maneira geral, é o caráter gregário das larvas que influencia a distribuição espacial agregada de carrapatos no ambiente. Em uma das subparcelas amostradas foram encontrados dois aglomerados de larvas, os quais representaram mais de $60 \%$ do número de carrapatos total do estudo. Uma vez que as larvas eclodem dos ovos, elas tendem a permanecer no mesmo lugar (Daniels \& Fish 1990) apresentando mínimos movimentos horizontais e um maior deslocamento vertical, quando sobem na vegetação para aumentar as chances de entrar em contato com hospedeiros (Szabó et al. 2009).

Na Mata Atlântica, grupos de carrapatos encontrados em alturas variáveis na vegetação podem permanecer mais de vinte e 24 na mesma posição à procura de hospedeiros. Ao contrário, em ambientes mais secos e ensolarados, se torna necessária uma migração verticalmente para baixo visando se proteger da dessecação no folhiço da mata. A capacidade de algumas espécies de ficar aguardando um hospedeiro por mais de vinte quatro horas só deve ser possível em ambientes mais sombreados, protegidos do sol (Szabó et al. 2009).

Foi observada correlação positiva entre a cobertura de dossel e a abundância de carrapatos nos trechos estudados. Isto sugere que os estágios imaturos de Amblyomma sp. não apresentam uma distribuição randômica e podem ser encontrados em áreas de mata mais sombreadas que são caracterizadas por fatores como maior umidade, menor temperatura e menor radiação solar, os quais são primordiais para a sobrevivência e diminuição do risco de dessecação dos carrapatos (Schulze et al. 2002, Schulze \& Jordan 2005). Em estudo realizado nesta área sobre a ocorrência agregada de Amblyomma fuscum no hospedeiro mais frequente localmente, o roedor Thrichomys laurentius, foi observado que os hospedeiros eram capturados em áreas mais abertas (Aléssio et al. 2012). Como sugere Leung (1998), a distribuição dos hospedeiros pode não influenciar diretamente a distribuição dos parasitos e o grau de agregação parece ser dependente de vários fatores, como a abundância média e a distância a qual os parasitos são capazes de detectar seus hospedeiros.

Além destes fatores, condições ambientais específicas produzidas pelo processo de fragmentação de habitats pa- recem ser capazes de influenciar a distribuição agregada de carrapato (Tack et al. 2012). No estudo realizado por Tack et al. (2012), foi observado uma maior abundância de carrapatos em fragmentos com maiores habitats de borda. Entretanto, o tipo de matriz que circunda as bordas de um determinado fragmento parece influenciar a abundância de carrapatos. No presente estudo foi observado que a maior abundância de carrapatos foi encontrada na região de borda da mata próximo à matriz agropastoril em relação à borda da mata próxima ao lago. Tais resultados correspondem ao padrão observado por Szabó et al. (2009) e Silveira \& Fonseca (2013), os quais encontram uma menor riqueza como também carrapatos pouco numerosos na borda de um fragmento que faz fronteira com barragem. Tal resultado pode ser importante, pois muitos hospedeiros de carrapatos, notadamente pequenos mamíferos, encontram também condições ideais de sobrevivência em área de borda de mata.

Deste modo, vertebrados terrestres (mamíferos, répteis e anfíbios) que habitam regiões de borda podem atuar como pontes epidemiológicas entre o fragmento e a matriz adjacente entre animais silvestres e domésticos que frequentam a matriz agropastoril (Boyard et al. 2008). Na área de estudo é comum encontrar gado pastando e se deslocando na matriz próxima à borda do fragmento. Tais animais podem transportar carrapatos para fora dos fragmentos estudados como também podem transportar novos carrapatos para ambientes de florestas. A borda da mata que margeia o lago é toda replantada de pau-brasil, Caesalpinia echina$t a$, o que pode ter dificultado a passagem da bandeira para a captura dos carrapatos por causa do grande número de espinhos nos troncos e nos brotos das plantas.

\section{CONCLUSÕES}

A distribuição espacial e a abundância predominantemente de larvas de Amblyomma sp. nas unidades de conservação de proteção integral estudadas são diretamente influenciadas pela cobertura do dossel e pela estrutura da matriz circundante dos fragmentos estudados.

Os carrapatos em Tapacurá apresentam uma dispersão agregada, o que confirma um padrão de distribuição espacial de carrapatos, a qual deve ser influenciada pelo comportamento gregário das larvas, que se mantém unidas após a eclosão dos ovos.

Em Tapacurá, foi encontrada uma abundância mais elevada de larvas na borda adjacente a uma região de matriz agropastoril.

Nessa região de transição podem ocorrer trocas de parasitos entre animais silvestres e domésticos, retroalimentando ciclos de transmissão de zoonoses.

Esforços futuros de amostragem devem expandir as áreas de estudo para as matrizes circundantes de habitats naturais para uma melhor compreensão das dinâmicas populacionais e também dos padrões de distribuição e a influência da estrutura de habitats na dispersão de carrapatos em estágio de vida livre.

\section{REFERÊNCIAS}

Aléssio F.M., Dantas-Torres F., Siqueira D.B., Lizée M.H., Marvulo M.F., Martins T.F., Labruna M.B., Silva J.C.R. \& Mauffrey J.F. 2012. Ecological impli- 
cations on the aggregation of Amblyomma fuscum (Acari: Ixodidae) on Thrichomys laurentius (Rodentia: Echimyidae), in northeastern Brazil. Exp. Appl. Acarol. 57:83-90.

Allan B.F., Keesing F. \& Ostfeld R.S. 2003. Effect of Forest Fragmentation on Lyme disease Risk. Conservation Biology 17(1):267-274.

Anderson J.F. \& Magnarelli L.A. 1993. Epizootiology of Lyme disease-causing borreliae. Clin. Dermatol. 11:339-351.

Aragão H.B. 1936. Ixodidas brasileiros e de alguns países limitrophes. Mem. Inst. Oswaldo Cruz 31(4):759-843.

Barreira J.D., Mares-Guia A.A.M.M. \& Moreira N.S. 2014. Procedimentos específicos para a coleta de carrapato, pulga, piolho e ácaros, p.137-141. In: Lemos E.R.S. \& D’Andrea P.S. (Eds), Trabalho de Campo com Animais: procedimentos, riscos e biossegurança. Fiocruz, Rio de Janeiro.

Boyard C., Vourc'h G. \& Barnouin J. 2008. The relationships between Ixodes ricinus and small mammal species at the woodland-pasture interface. Exp. Appl. Acarol. 44(1): 61-76.

Condepe 2000. Base de dados do Estado, Climatologia: descrição dos tipos. Governo do Estado de Pernambuco, Instituto de Planejamento de Pernambuco, Recife.

Daniels T.J. \& Fish D. 1990. Spatial distribution and dispersal of unfed larval Ixodes dammini (Acari: Ixodidae) in southern New York. Environ. Entomol. 19(4):1029-1033.

Dantas-Torres F., Aléssio F.M., Siqueira D.B., Mauffrey J.F., Marvulo M.F.V., Martins T.F., Moraes-Filho J., Camargo M.C.G.O., D’Auria S.R.N., Labruna M.B. \& Silva J.C.R. 2012. Exposure of small mammals to ticks and rickettsiae in Atlantic Forest patches in the metropolitan area of Recife, North-eastern Brazil. Parasitology 139(1):83-91.

Dantas-Torres F., Oliveira-Filho E.F., Soares F.A.M., Souza B.O.F., Valença R.B.P. \& Sá F.B. 2008. Ticks infesting amphibians and reptiles in Pernambuco, Northeastern Brazil. Revta Bras. Parasitol. Vet. 17(4):218-221.

Dantas-Torres F., Onofrio V.C. \& Barros-Battesti D.M. 2009. The ticks (Acari: Ixodida: Argasidae, Ixodidae) of Brazil. System. Appl. Acarol. 14:30-46.

Estrada-Peña A. 2001. Distribution, abundance, and habitat preferences of Ixodes ricinus (Acari: Ixodidae) in Northern Spain. J. Med. Entomol. 38:361-370.

Freitas S.R., Cerqueira R. \& Vieira M.V. 2002. Adevice and standard variables to describe microhabitat structure of small mammals based on plant cover. Braz. J. Biol. 62(4B):795-800.

Galindo-Leal C. \& Câmara I.G. 2003. The Atlantic Forest of South America: biodiversity status, threats, and outlook. Island Press, Washington, DC, USA. 488p.

Guerra M., Walker E., Jones C., Paskewitz S., Cortinas M.R., Stancil A., Beck L., Bobo M. \& Kitron U. 2002. Predicting the risk of lyme disease: habitat suitability for Ixodes scapularis in the North Central United States. Emerg. Infect. Dis. 8:289-297.

Horta M.C., Nascimento G.F., Martins T.F., Labruna M.B., Machado L.C.P. \& Nicola P.A. 2011. Ticks (Acari: Ixodida) parasitizing free-living wild animals in the Caatinga biome in the State of Pernambuco, northeastern Brazil. System. Appl. Acarol. 16(3):207-211.

Labruna M.B., Camargo L.M., Terrassini F.A., Ferreira F., Schumaker T.S. \& Camargo E.P. 2005. Ticks (Acari: Ixodidae) from the state of Rondônia, Western Amazon, Brazil. System. Appl. Acarol. 10(1):17-32.

Leung B. 1998. Aggregated parasite distributions on hosts in a homogeneous environment: examining the Poisson null model. Int. J. Parasitol. 28:1709-1712.

Lindgren E., Talleklint L. \& Polfeldt T. 2000. Impact of climatic change on the northern latitude limit and populations density of the disease-transmitting European tick Ixodes ricinus. Environ. Health Perspect. 108:119-123.

Lyra-Neves R.M., Oliveira M.A.B., Telino Júnior W.R. \& Santos E.M. 2007. Comportamentos interespecíficos entre Callithryx jacchus (Linnaeus) (Primates; Callitrichidae) e algumas aves de Mata Atlântica, Pernambuco, Brasil. Revta Bras. Zool. 3(24):709-716.

Martins T.F., Onofrio V.C., Barros-Battesti D.M. \& Labruna M.B. 2010. Nymphs of the genus Amblyomma (Acari: Ixodidae) of Brazil: descrip- tions, redescriptions, and identification key. Ticks Tick Borne Dis. 1(2):75-99.

Martins T.F., Venzal J.M., Terassini F.A., Costa F.B., Marcili A., Camargo L.M., Barros-Battesti D.M. \& Labruna M.B. 2014. New tick records from the state of Rondônia, western Amazon, Brazil. Exp. Appl. Acarol. 62(1):121128.

Murcia C. 1995. Edge effects in fragmented forests: implications for conservation. Trends Ecol. Evol. 10:58-62.

Nava S., Beati L., Labruna M.B., Cáceres A.G., Mangold A.J. \& Guglielmone A.A. 2014. Reassessment of the taxonomic status of Amblyomma cajennense Fabricius, 1787 with the description of three new species, Amblyomma tonelliae n. sp., Amblyomma interandinum n. sp. and Amblyomma patinoi n. sp., and reinstatement of Amblyomma mixtum Koch, 1844 and Amblyomma sculptum Berlese, 1888 (Ixodida: Ixodidae). Ticks Tick Borne Dis. 5(3):252-276.

Nava S., Mangold A.J. \& Guglielmone A.A. 2009. Seasonal distribution of larvae and nymphs of Amblyomma tigrinum Koch, 1844 (Acari: Ixodidae). Vet. Parasitol. 166:340-342.

Onofrio V.C., Labruna M.B., Pinter A., Giacomin F.G. \& Barros-Battesti D. 2006. Comentários e chaves para as espécies do gênero Amblyomma, p. 53-113. In: Barros-Battesti D.M., Arzua M. \& Bechara G.H. (Eds), Carrapatos de Importância Médico-veterinária da Região Neotropical: um guia ilustrado para identificação de espécies. Integrated Consortium on Ticks and Tick-borne Diseases (ICTTD), Vox, Butantan, São Paulo.

Onofrio V.C. 2007. Revisão do gênero Amblyomma Koch, 1844 (Acari: Ixodidae) no Brasil. Tese de Doutorado, Ciências Veterinárias, Universidade Federal Rural do Rio de Janeiro, Seropédica, Rio de Janeiro. 174p.

Ostfeld R.S., Miller M.C. \& Hazler K.R. 1996. Causes and consequences of tick (Ixodes scapularis) burdens on white-footed mice (Peromyscus leucopus). J. Mammalogy 77(1): 266-273.

Pernambuco 2011. Lei no. 14.324, de 03 junho de 2011, Categoriza as Reservas Ecológicas da Região Metropolitana do Recife, e dá outras providências. Diário Oficial do Estado de Pernambuco, Poder Executivo Recife. Acesso em 4 jun/2011.

Peterka C.R.L. 2008. Avaliação do efeito da fragmentação florestal na diversidade de carrapatos e patógenos transmitidos por carrapatos na região do Pontal do Paranapanema, SP. Dissertação de Mestrado, Medicina Veterinária, Universidade de São Paulo, São Paulo.

Pinheiro M.C., Lourenço E.C., Patrício P.M.P., Sá-Hungaro I.J.B. \& Famadas K.M. 2014. Free-living ixodid ticks in an urban Atlantic Forest fragment, state of Rio de Janeiro, Brazil. Revta Bras. Parasitol. Vet. 23(2):264-268.

Ramos V.N., Osava C.F., Piovezan U. \& Szabó M.P.J. 2014. Complementary data on four methods for sampling free-living ticks in the Brazilian Pantanal. Braz. J. Vet. Parasitol. 23(4):516-521.

Randolph S.E. \& Storey K. 1999. Impact of microclimate on immature tick-rodent interactions (Acari: Ixodidae): implications for parasite transmission. J. Med. Entomol. 36:741-748.

Rodrigues E. 1998. Edge Effects on the regeneration of forest fragments in South Brasil. Thesis. Harvard University.

Rosenberg M.S. \& Anderson C.D. 2011. PASSaGE: Pattern Analysis, Spatial Statistics and Geographic Exegesis. Version 2. Methods Ecol. Evol. 2(3):229-232.

Rózsa L., Reiczigel J. \& Majoros G. 2000. Quantifying parasites in samples of hosts. J. Parasitol. 86:228-232.

Rulison E.L., Kuczaj I., Pang G., Hickling G.J., Tsao J.I., Howard S. \& Ginsberg H.S. 2013. Flagging versus dragging as sampling methods for nymphal Ixodes scapularis (Acari: Ixodidae). J. Vector Ecol. 38(1):163-167.

Santos E.M., Botelho M.C.N. \& De Oliveira J.B. 2002. Ectoparasitos de anfíbios anuros (Anura, Bufonidae) capturados na Estação Ecológica do Tapacurá, São Lourenço da Mata, Pernambuco, Brasil. Entomol. Vectores 9(1):105-113.

Schulze T.L., Jordan R.A. \& Hung R.W. 2002. Effects of microscale habitat physiognomy on the focal distribution of Ixodes scapularis and Amblyomma americanum (Acari: Ixodidae) nymphs. Environmental Entomol. 31(6):1085-1090.

Schulze T.L. \& Jordan R.A. 2005. Influence of Meso- and Microscale Habitat 
Structure on Focal Distribution of Sympatric Ixodes scapularis and Amblyomma americanum (Acari: Ixodidae). J. Med. Entomol. 42(3):285-294.

Schumaker T.T.S. \& Barros D.M. 1994. Notes on the biology of Amblyomma dissimile Koch, 1844 (Acari: Ixodidae) on Bufo marinus (Linnaeus, 1758) from Brazil. Mem. Inst. Oswaldo Cruz 89(1):29-31.

Silvano D.L. \& Segala M.V. 2005. Conservação de anfíbios no Brasil. Megadiversidade 1(1):79-86.

Silveira A.K. \& Fonseca A.H. 2013. Distribuição, diversidade e sazonalidade de carrapatos em ambientes institucionais com diferentes graus de intervenção humana no Estado do Rio de Janeiro, Brasil. Revta Bras. Med. Vet. 35:1-12.

Silveira I., Pacheco R.C., Szabó M.P., Ramos H.G. \& Labruna M.B. 2007. Rickettsia parkeri in Brazil. Emerg. Infect. Dis. 13:1111-1113.

Sonenshine D.E., Nicholson W.L. \& Lane R.S. 2002. Ticks (Ixodidae), p.517558. In: Mullen G. \& Durden L. (Eds), Medical and Veterinary Entomology. Academic Press Elsevier Science, Amsterdam.
Spolidorio M.G., Labruna M.B., Mantovani E., Brandao P.E., Richtzenhain L.J. \& Yoshinari N.H. 2010. Novel spotted fever group rickettsiosis, Brazil. Emerg. Infect. Dis. 16:521-523.

Szabó M.P.J., Castro M.B., Ramos H.G, Garcia M.V., Castagnolli K.C., Pinter A., Veronez V.A., Magalhães G.M., Duarte G.M. \& Labruna M.B. 2007. Species diversity and seasonality of free-living ticks (Acari: Ixodidae) in the natural habitat of wild Marsh deer (Blastocerus dichotomus) in southeastern Brazil. Vet. Parasitol. 143(2):147-154.

Szabó M.P.J., Labruna M.B., Garcia M.V., Pinter A., Castagnolli K.C., Pacheco R.C., Castro M.B., Veronez V.A., Magalhães G.M., Vogliotti A. \& Duarte J.M.B. 2009. Ecological aspects of the free-living ticks (Acari: Ixodidae) on animal trails within Atlantic rainforest in south-eastern Brazil. Annu. Trop. Med. Parasitol. 103(1):57-72.

Tack W., Madder M., Baeten L., Vanhellemont M., Gruwez R. \& Verheyen K. 2012. Local habitat and landscape affect Ixodes ricinus tick abundances in forests on poor, sandy soils. Forest Ecol. Management 265:30-36. 